


\title{
Perspectivas temporales en las actividades profesionales de especialistas en el área económica
}

\author{
DOI: https://doi.org/10.46398/cuestpol.3969.27
}

\author{
Ihor Popovych * \\ Diego Felipe Arbeláez-Campillo ** \\ Magda Julissa Rojas-Bahamón *** \\ Iryna Burlakova **** \\ Vitaliy Kobets ***** \\ Halyna Bokshan ******
}

\section{Resumen}

El propósito de la investigación es estudiar las perspectivas temporales en las actividades profesionales de los especialistas en el área económica. Es sabido que los especialistas con más de veinte años de experiencia se centran principalmente en el pasado y el presente, y los que acumulan menos de diez años, en el futuro. La base metodológica de la investigación fue el concepto de perspectiva temporal. Esta metodología ha sido aprobada por investigadores en el estudio de los trastornos de adaptación, ansiedad, innovación. Está demostrado que los representantes del grupo profesional de especialistas que acumulan menos de diez años de experiencia están más inclinados a la planificación estratégica y a la busca del apoyo emocional en situaciones difíciles, y que los especialistas con 10-20 años de experiencia, a la superación proactiva y reflexiva. Se ha descubierto también que los hombres tienen una orientación temporal más pronunciada para el "Futuro", y las mujeres, para el "Futuro trascendental" $(\mathrm{p} \leq .05)$. Las mujeres son más propensas a la inmersión emocional en los eventos actuales, mientras que los hombres son más

* Doctor of Psychological Sciences, Full Professor of Department of Psychology, Kherson State University, Kherson, Ukraine. ORCID ID: https://orcid.org/oooo-0002-1663-111X.

** Grupo de Investigación Lenguajes, Representaciones y Educación, Universidad de la Amazonia, Colombia. ORCID ID: https://orcid.org/0000-0002-9041-9563.

*** PhD. in Education and environmental Culture. Professor Jorge Eliecer Gaitán and Universidad de la Amazonia, Colombia. ORCID ID: https://orcid.org/oooo-0oo3-4882-1476.

**** Doctor of Psychological Sciences, Professor of Department of Practical Psychology and Social Work, Volodymyr Dahl East-Ukrainian National University, Severodonetsk, Ukraine. ORCID ID: https:// orcid.org/oooo-0002-6043-4359.

**** Doctor of Economic Sciences, Professor of Department of Computer Science and Economic Cybernetics, Kherson State University, Kherson, Ukraine. ORCID ID: https://orcid.org/oooo-00024386-4103.

****** PhD., Associate Professor of Department of Tourism, Hotel and Restaurants Business and Foreign Languages, Kherson State Agrarian and Economic University, Kherson, Ukraine. ORCID ID: https:// orcid.org/oooo-0002-7430-8257. 
propensos a estructurar los eventos actuales y planificar eventos futuros. ( $\mathrm{p} \leq .05)$. Es aconsejable utilizar los resultados obtenidos en asesoramiento psicológico y evaluación del personal.

Palabras clave: tiempo; comportamiento; género; futuro trascendental; economista.

\title{
Time perspective in the professional activity of specialists of economic sphere
}

\begin{abstract}
The aim of the article is the research of time perspectives in the professional activity of specialists of economic sphere. It is established that specialists with experience more than twenty years have largely focused on past and present, and specialists with experience of up to ten years on the future. The methodological basis of the research was the concept of time perspective. This methodology has been approved by researchers in the study of adaptation disorders, anxiety, innovation. The representatives of the professional group of specialists with experience up to ten years may be more tend to strategic planning and the search for emotional support in difficult situations; and specialists with experience of 10-20 years - to a proactive and reflexive overcome. It is found that men are more express for temporal focus of the "Future," and women - for "Transcendent future" $(\mathrm{p} \leq .05)$. Women have a more expressed tendency to emotional immersion in the events of the present, and men - structuring of current events and planning future events $(\mathrm{p} \leq .05)$. The received results should be used in psychological counseling and estimation of staff.
\end{abstract}

Key words: time; behavior; gender; transcendent future; economist.

\section{Introducción}

La dinámica negativa de los principales indicadores financieros y económicos del país, la desestabilización del mercado laboral, la creciente tensión social y la disminución de los niveles de vida no podían sino afectar al individuo. La alteración de un sistema económico estable ha llevado a cambios en el espacio mental del individuo, y también en el sistema de autoconciencia del individuo.

Un componente extremadamente importante del bienestar psicológico de una persona es la capacidad de determinar independientemente los 
Ihor Popovych, Diego Felipe Arbeláez-Campillo, Magda Julissa Rojas-Bahamón, Iryna Burlakova, Vitaliy Kobets y Halyna Bokshan

objetivos de su vida (Blynova y Kruglov, 2019; Blynova et al., 2020b; 2020c). Esto se debe a la presencia en la imagen del mundo del individuo de una perspectiva temporal del futuro larga y rica en contenido. El tiempo es uno de los factores importantes, pero rara vez se utiliza para las reservas de organización mental del individuo, para su autorrealización en la sociedad. Las perspectivas de tiempo del individuo pueden verse como manifestaciones que regulan las posibilidades y la elección de estrategias del comportamiento en situaciones difíciles. La formación de una perspectiva temporal se convierte en un medio de autorregulación, una forma de desarrollo de las estrategias de comportamiento en situaciones difíciles de la vida.

\section{Revisión de la literatura}

En la psicología moderna, todavía no existe una comprensión única del término "perspectiva de tiempo o perspectiva temporal": varios científicos al estudiar este fenómeno enfatizan uno de los tres aspectos. El primer aspecto es según Nuttin (2004): "La perspectiva de tiempo en el sentido propio de la palabra se caracteriza principalmente por la longitud, profundidad, saturación, grado de estructuración y nivel del realismo" (2004: 354). Esta opinión es sostenida por J. Nuttin (2004). Él dice que los actos de comportamiento obviamente están sucediendo hoy bajo la influencia de las circunstancias actuales, pero no debemos olvidar que el comportamiento también está determinado por eventos pasados y futuros (Arbeláez-Campillo et al., 2018; Blynova et al., 2020a; 2020d). Los psicólogos hablan constantemente de la influencia del pasado, en particular de la formación, la experiencia, pero no ignoran tampoco la importancia de los eventos futuros: expectativas, predicciones, anticipación (Kobets y Poltoratskiy, 2014; Kobets y Yatsenko, 2016; Plokhikh, 2006).

Hay disputas acerca de que las expectativas y las predicciones se basan de una u otra manera en la experiencia previa del sujeto (Kononenko et al., 2020; Pinkovetskaia et al., 2020). En consecuencia, J. Nuttin (2004) en su concepto enfatiza lo siguiente: "El pasado no tiene nada que ver con el futuro. $<\ldots>$ la orientación hacia el futuro es un fenómeno nuevo y peculiar que surge en presencia de un estado motivacional" (2004: 358).

En el ser humano, como en un ser que tiene funciones cognitivas más altas y una transformación cognitiva de las necesidades en objetivos, la predicción se aleja de la situación actual, formando una perspectiva temporal cada vez más profunda. En la descripción de la perspectiva temporal se enfatiza (Nuttin, 2004): "Su componente "material" ("objetivo") son los objetos del pasado y del futuro", lo que constituye su contenido" (2004; 359). El segundo aspecto destaca según Prokonich (2014): "El entorno 
temporal, es decir, el estado de ánimo más o menos positivo o negativo del sujeto hacia el pasado, presente o futuro" (2014: 118).

La idea principal de este enfoque es que la actitud del sujeto hacia la perspectiva temporal del pasado, presente o futuro está influenciada por sus características personales, así como por la experiencia de interacción con diferentes objetos en diferentes situaciones, lo que afecta su evaluación del entorno en su conjunto y en algunos de sus aspectos (Prokonich, 2014).

Por su parte, El tercer aspecto según Nuttin:

La orientación temporal caracteriza el comportamiento del sujeto y, por lo tanto, se considera como el enfoque predominante de este comportamiento en objetos y eventos del pasado, presente o futuro. Por ejemplo, se cree que los jóvenes están más orientados hacia el futuro y las personas mayores están más centradas en el pasado (2004: 360).

Basándonos en este criterio, hemos estudiado las características de la perspectiva de tiempo y la estructura de su relación con los indicadores de autorregulación del comportamiento de las personas en diferentes períodos de la edad adulta. Los investigadores de este problema afirman que diferentes perspectivas de tiempo tienen diferentes significados a diferentes edades. En particular, al final de la edad adulta, a la edad avanzada, el futuro es incierto e incluye el miedo al cambio para peor y a la muerte próxima (Shul'c y Shul'c, 2002). En cuanto al pasado, se puede notar que la madurez se caracteriza por contar los eventos desde la infancia, luego en la vejez este punto ya está al comienzo de la actividad profesional (Shul'c y Shul'c, 2002).

Uno de los puntos de vista más modernos sobre la perspectiva temporal del individuo, perteneciente a F. Zimbardo y D. Bojd, es atractivo. Definen la perspectiva temporal del individuo de la siguiente manera: "A menudo, la actitud inconsciente de una persona hacia el tiempo es un proceso mediante el cual el largo flujo de existencia se combina en categorías de tiempo, lo que ayuda a organizar nuestra vida, estructurarla y darle significado" (2010:12).

Esta concepción es interesante desde el punto de vista de combinar el segundo y el tercer aspecto del concepto de perspectiva temporal: los tipos seleccionados de perspectiva temporal reflejan claramente la actitud positiva o negativa de la persona hacia lo más importante para ella: tiempo pasado, presente o futuro; así como la descripción de los tipos de perspectivas temporales se presenta directamente a través de la descripción del comportamiento de la persona. En este sentido, F. Zimbardo y D. Bojd (2010) consideran la perspectiva temporal de los profesionales económicos en términos del segundo y tercer aspecto. 
Ihor Popovych, Diego Felipe Arbeláez-Campillo, Magda Julissa Rojas-Bahamón, Iryna Burlakova, Vitaliy Kobets y Halyna Bokshan

La investigación organizada está dirigida al estudio de las características de la perspectiva temporal de los indicadores de actividad profesional en especialistas de la esfera económica. Los especialistas en la esfera económica son oficinistas, cuyo trabajo se acompaña en parte de una gran multifuncionalidad y varias sobrecargas psicoemocionales (Allen, 2002; Bolotova, 2012; Kalenchuk, 2019; Khmiliar et al., 2020; Krupnyk y Tkalenko, 2019). La hipótesis principal de nuestro estudio es la suposición de que los indicadores de la perspectiva de tiempo están interrelacionados con los indicadores de actividad profesional de los especialistas económicos. El propósito de nuestro estudio es establecer perspectivas temporales en las actividades profesionales de los especialistas económicos.

\section{Metodología}

La base metodológica de la investigación organizada en el contexto del establecimiento de perspectivas temporales en la actividad profesional de especialistas en la esfera económica es el complejo metodológico aprobado con el uso de los instrumentos de psicodiagnóstico (Blynova et al., 2020b; Fernández-Rivas y Espada-Mateos, 2019; Moya et al., 2018; Popovych et al., 2020a; Shevchenko et al., 2020). Esta metodología ha sido aprobada por investigadores en el estudio de los trastornos de adaptación, ansiedad, innovación (Acevedo-Duque et al., 2020; Halian et al., 2020a; 2020b; 2020c; Varguillas y Bravo, 2020), así como en el estudio de las expectativas mentales en diversas actividades de los encuestados (Cruz, 2019; Nosov et al., 2020a; 2020b; Romero-Argueta et al., 2020; Tsiuniak et al., 2020; Zinchenko et al., 2019; 2020). Todas estas mediciones experimentales y empíricas son relevantes para las perspectivas temporales en las actividades profesionales de los especialistas económicos.

Participantes. La muestra ha estado compuesta por 112 futuros especialistas en la esfera económica, que recibían la formación profesional en las facultades de economía, economía y finanzas, economía internacional, gestión de personal y economía laboral. También 126 especialistas en la esfera económica, a saber, empleados bancarios, analistas financieros, economistas de negocios, gerentes de proyectos y programas de inversión, gerentes con diferente experiencia profesional. La edad promedio de los sujetos fue de 31.5 años (SD = 6.11). El grupo profesional de empleados que trabajan con clientes internos estaba formado por administradores, asistentes, gerentes, en total 48 personas de las cuales 26 hombres y 22 mujeres. El grupo profesional de empleados que trabajan con clientes externos estaba formado por gerentes de ventas, gerentes de servicio al cliente, consultores, en total 44 personas de las cuales 25 hombres y 19 mujeres. En el grupo profesional de gerentes, desde el jefe del departamento hasta el CEO de una empresa mediana, había 34 participantes: 18 hombres 
y 16 mujeres. La investigación fue realizada de acuerdo con las normas éticas del comité sobre los derechos de los experimentos de Declaración de Helsinki (JAMA Network 2013).

Procedimientoyinstrumentos. Siguiendo el concepto deperspectiva temporal de F. Zimbardo y D. Bojd (2010), utilizamos los métodos "Zimbardo Time Perspective Inventory" ("ZTPI") (Zimbardo y Bojd, 2010). "Transcendental-future Time Perspective Inventory" ("TFTPI") (Zimbardo y Bojd, 2010). También usamos el "Método Diferencial Semántico del Tiempo" (“SDT”) (Vasserman et al., 2009).

“Zimbardo Time Perspective Inventory" (“ZTPI”) se publicó en 1997, y "Transcendental-future Time Perspective Inventory" (“TFTPI") - en 1999. Más tarde ellos fueron adaptados por eminentes científicos. Estos cuestionarios consisten en 56 y 10 ítems, respectivamente, y están destinados a establecer perspectivas temporales del individuo tales como: pasado negativo, pasado positivo, presente fatalista, presente hedonista, futuro y futuro trascendente. Se usó una escala de cinco puntos. Los puntos en cada escala se suman y se dividen por el número de declaraciones relacionadas con una perspectiva temporal dada. Entonces, para cada perspectiva de tiempo, el "puntaje promedio" se calcula en el intervalo de 1.00 a 5.00 (Zimbardo y Bojd, 2010). Por lo tanto: en los indicadores de 1.00 a 2.50, la perspectiva de tiempo dada no se expresa; de 2.50 a 3.75 es el grado promedio de la perspectiva temporal; los puntajes de 3.75 a 5.00 indican que esta perspectiva de tiempo es más pronunciada, y con mayor frecuencia es principal en el encuestado. El método "Diferencial semántico del tiempo" ("SDT") se compone de tres impresos idénticos (con instrucciones para el presente, pasado y futuro). Sobre la base de veinticinco pares de adjetivos, se identifican cinco factores: actividad del tiempo (AT), coloración emocional del tiempo (CET), magnitud del tiempo (MT), estructura del tiempo (ET) y sentido del tiempo (ST). Es aconsejable determinar el valor promedio de la evaluación (VPE) de cada momento: pasado, presente y futuro (Vasserman et al., 2009).

Análisis estadístico. El procesamiento de datos estadísticos y la presentación gráfica de los resultados de la investigación se llevaron a cabo utilizando un paquete de programas estadísticos "Statistical Package for the Social Sciences" v. 21.0 y "Microsoft Office Excel 2007". Para sistematizar los datos obtenidos y amplificar las variables se utilizó el análisis factorial. Esto nos permitió identificar estructuras de investigación más globales las que se encuentran fuera de las dimensiones empíricas de estas técnicas. 
Ihor Popovych, Diego Felipe Arbeláez-Campillo, Magda Julissa Rojas-Bahamón, Iryna Burlakova, Vitaliy Kobets y Halyna Bokshan

430

Perspectivas temporales en las actividades profesionales de especialistas en el área económica

\section{Resultados}

Vamos a considerar las características de las perspectivas temporales de acuerdo con "Zimbardo Time Perspective Inventory" ("ZTPI") y "Transcendental-future Time Perspective Inventory" ("TFTPI") en los representantes de diversos grupos profesionales de especialistas en la esfera económica (Tabla 1).

Tabla 1. Indicadores de perspectivas temporales en diversos grupos profesionales de especialistas en el área económica de acuerdo con "ZTPI" y “TFTPI”

\begin{tabular}{|c|c|c|c|c|c|c|c|}
\hline \multirow{2}{*}{ Escala } & & \multicolumn{6}{|c|}{ Parámetros } \\
\hline & & \multirow{2}{*}{ 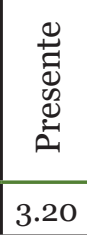 } & \multirow{2}{*}{ 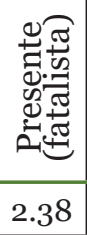 } & \multirow{2}{*}{ 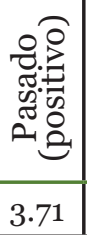 } & \multirow{2}{*}{ 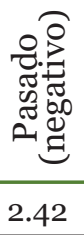 } & \multirow{2}{*}{$\begin{array}{l}\stackrel{0}{\stackrel{\Xi}{\Xi}} \\
3.68\end{array}$} & \multirow{2}{*}{ 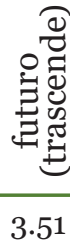 } \\
\hline \multirow{2}{*}{$\begin{array}{l}\text { Desarrollo de la conciencia } \\
\text { profesional }\end{array}$} & M & & & & & & \\
\hline & SD & .43 & .53 & .47 & .50 & .45 & .71 \\
\hline \multirow{2}{*}{$\begin{array}{l}\text { Especialistas con menos de } 10 \\
\text { años de experiencia }\end{array}$} & $\mathrm{M}$ & 3.07 & 2.44 & 3.79 & 2.57 & 3.91 & 3.37 \\
\hline & SD & .32 & .56 & .49 & .53 & .48 & .78 \\
\hline \multirow{2}{*}{$\begin{array}{l}\text { Especialistas con 10-20 años } \\
\text { de experiencia }\end{array}$} & M & 3.22 & 2.39 & 3.70 & 2.47 & 3.62 & 3.53 \\
\hline & SD & .49 & .59 & .47 & .47 & .45 & .74 \\
\hline \multirow{2}{*}{$\begin{array}{l}\text { Especialistas con más de } 20 \\
\text { años de experiencia }\end{array}$} & $\mathrm{M}$ & 3.28 & 2.29 & 3.66 & 2.17 & 3.61 & 3.60 \\
\hline & SD & .33 & .33 & .44 & .46 & .37 & .57 \\
\hline \multirow{2}{*}{$\begin{array}{l}\text { Diferencias significativas } \\
\text { entre grupos: Especialistas } \\
\text { con menos de } 10 \text { años de } \\
\text { experiencia y Especialistas } \\
\text { con 10-20 anos de experiencia }\end{array}$} & $\mathrm{F}$ & - & - & - & - & .29 & - \\
\hline & $\varphi$ & - & - & - & - & .023 & - \\
\hline \multirow{2}{*}{$\begin{array}{l}\text { Diferencias significativas } \\
\text { entre grupos: Especialistas } \\
\text { con menos de } 10 \text { años de } \\
\text { experiencia y Especialistas } \\
\text { con más de } 20 \text { anos de } \\
\text { experiencia }\end{array}$} & $\mathrm{F}$ & - & - & - & .4 & $\cdot 3$ & - \\
\hline & $\varphi$ & - & - & - & .014 & .049 & \\
\hline
\end{tabular}




\begin{tabular}{|l|l|l|l|l|l|l|l|}
\hline $\begin{array}{l}\text { Diferencias significativas } \\
\text { entre grupos:Especialistas } \\
\text { con 10-20 años de experiencia }\end{array}$ & F & - & - & - & .3 & - & - \\
\cline { 2 - 8 } $\begin{array}{l}\text { Especialistas con más de 20 } \\
\text { años de experiencia }\end{array}$ & $\varphi$ & - & - & - &. $\mathbf{0 2 7}$ & - & - \\
\hline
\end{tabular}

Fuente: Elaboración propia, 2021.

Nota: M - media aritmética; SD - desviación de la media cuadrada; F - la diferencia promedio; $\varphi$ valor; destacamos diferencias significativas en negrita y diferencias a nivel de la tendencia estadística en cursiva.

Se ha establecido que los indicadores de las perspectivas de tiempo en los especialistas que se encuentran en la etapa de desarrollo de la conciencia profesional de nuestra muestra están en el intervalo de valor promedio. Las puntuaciones más altas se observan en las escalas de las perspectivas temporales del pasado y futuro positivo, y las más bajas, en las escalas del pasado fatalista verdadero y pasado negativo. En el grupo profesional de encuestados "Especialistas con menos de 10 años de experiencia", las orientaciones temporales más pronunciadas son para el futuro, el pasado positivo y el futuro trascendente. En los grupos profesionales "Especialistas con 10-20 años de experiencia" y "Especialistas con más de 20 años de experiencia", la atención se centra en el pasado positivo, así como en el futuro más pronunciado y el futuro trascendente. Entonces, de acuerdo con la estructura de las perspectivas temporales, los grupos profesionales de encuestados no tienen diferencias significativas.

Se han encontrado también diferencias significativas $(\mathrm{p}<.05)$ entre los representantes de grupos profesionales de encuestados "Especialistas con menos de 10 años de experiencia" y "Especialistas con más de 20 años de experiencia", así como "Especialistas con experiencia de 10 a 20 años" y "Especialistas con experiencia de más de 20 años" en la escala de "Pasado negativo": en ambos casos, los indicadores son inferiores en los especialistas con más de 20 años de experiencia. Es decir, los encuestados con más de 20 años de experiencia son menos propensos que aquellos con menos de 20 años de experiencia a ser pesimistas sobre su pasado. Los resultados de la escala "Futuro" son más altos para los especialistas con menos de 10 años de experiencia. Por lo tanto, los representantes de este grupo profesional son más propensos a planificar su futuro que los representantes de los otros tres grupos.

Conviene considerar además las características de las perspectivas temporales de acuerdo con "Zimbardo Time Perspective Inventory" ("ZTPI") y "Transcendental-future Time Perspective Inventory" ("TFTPI") para representantes de varios grupos profesionales de especialistas económicos, teniendo en cuenta el género de los encuestados (Tabla 2). 
Ihor Popovych, Diego Felipe Arbeláez-Campillo, Magda Julissa Rojas-Bahamón, Iryna Burlakova, Vitaliy Kobets y Halyna Bokshan

Tabla 2. Indicadores de perspectivas temporales en diversos grupos profesionales de especialistas en el área económica, teniendo en cuenta el factor de género de acuerdo con "ZTPI" y “TFTPI"

\begin{tabular}{|l|c|c|c|c|l|l|}
\hline \multirow{2}{*}{ Escala } & \multicolumn{2}{|l|}{ Hombres } & \multicolumn{2}{l|}{ Mujeres } & \multicolumn{2}{l|}{$\begin{array}{l}\text { Valores de las } \\
\text { diferencias }\end{array}$} \\
\cline { 2 - 8 } & $\mathrm{M}$ & $\mathrm{SD}$ & $\mathrm{M}$ & $\mathrm{SD}$ & $\mathrm{F}$ & $\boldsymbol{\varphi}$ \\
\hline Presente & 3.27 & .42 & 3.14 & .44 & - & - \\
\hline Presente (fatalista) & 2.28 & .56 & 2.47 & .50 & - & - \\
\hline Pasado (positivo) & 3.61 & .50 & 3.79 & .42 & - & - \\
\hline Pasado (negativo) & 2.39 & .42 & 2.44 & .56 & - & - \\
\hline Futuro & 3.82 & .42 & 3.55 & .44 & $\mathbf{7 . 6 9}$ & $\mathbf{. 0 0 7}$ \\
\hline Futuro (trascendente) & 3.27 & .76 & 3.73 & .58 & $\mathbf{1 1 . 6 4}$ & $\mathbf{. 0 0 1}$ \\
\hline
\end{tabular}

Fuente: Elaboración propia, 2021.

Nota: $\mathrm{M}$ - media aritmética; SD - desviación de la media cuadrada; F - la diferencia promedio; $\varphi$ valor; destacamos diferencias significativas en negrita y diferencias a nivel de la tendencia estadística en cursiva.

El análisis de varianza ha revelado diferencias significativas $(\mathrm{p}<.01)$ entre hombres y mujeres en las escalas: "Futuro" (más pronunciado en hombres) y "Futuro trascendente" (más pronunciado en mujeres). Los hombres están más inclinados que las mujeres a planificar cuidadosamente su futuro, a establecer metas y planes para alcanzar. Al mismo tiempo, las mujeres son más religiosas que los hombres, creen en la vida después de la muerte.

Percepción subjetiva del tiempo en diferentes grupos profesionales de especialistas en el área económico, teniendo en cuenta el factor de género por el método de DST. Vamos a considerar las características de la percepción subjetiva del pasado en representantes de diferentes grupos profesionales por el método del "Diferencial Semántico del Tiempo" ("DST") (ver Tabla $3)$. 
Tabla 3. Indicadores de percepción subjetiva del pasado en diversos grupos profesionales de especialistas en el área económica de acuerdo con "SDT"

\begin{tabular}{|c|c|c|c|c|c|c|c|}
\hline \multirow{2}{*}{ Escala } & & \multicolumn{6}{|c|}{ Parámetros } \\
\hline & & 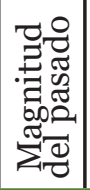 & 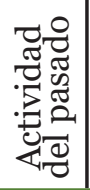 & 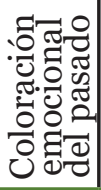 & 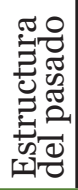 & 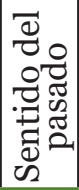 & 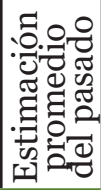 \\
\hline \multirow{2}{*}{$\begin{array}{l}\text { Desarrollo de la conciencia } \\
\text { profesional }\end{array}$} & M & 6.35 & 3.87 & 7.70 & 1.42 & 1.70 & 4.21 \\
\hline & $\mathrm{SD}$ & 5.25 & 5.27 & 5.09 & 5.09 & 4.81 & 3.75 \\
\hline \multirow{2}{*}{$\begin{array}{l}\text { Especialistas con menos de } 10 \\
\text { años de experiencia }\end{array}$} & M & $5 \cdot 39$ & 3.87 & 7.91 & 1.70 & .91 & 3.96 \\
\hline & SD & 5.84 & 6.06 & 3.09 & 4.74 & 4.30 & 3.04 \\
\hline \multirow{2}{*}{$\begin{array}{l}\text { Especialistas con 10-20 años } \\
\text { de experiencia }\end{array}$} & M & 5.63 & 2.92 & 6.63 & .94 & 1.87 & 3.60 \\
\hline & $\mathrm{SD}$ & $5 \cdot 33$ & $5 \cdot 54$ & 5.15 & 4.71 & 4.69 & 3.81 \\
\hline \multirow{2}{*}{$\begin{array}{l}\text { Especialistas con más de } 20 \\
\text { años de experiencia }\end{array}$} & M & 8.75 & 5.96 & 9.89 & 2.29 & 1.96 & $5 \cdot 77$ \\
\hline & $\mathrm{SD}$ & 3.79 & 3.09 & 5.67 & 6.13 & 5.53 & 3.83 \\
\hline \multirow{2}{*}{$\begin{array}{l}\text { Diferencias significativas } \\
\text { entre grupos: Especialistas } \\
\text { con menos de } 10 \text { años de } \\
\text { experiencia y Especialistas con } \\
\text { 10-20 años de experiencia }\end{array}$} & $\mathrm{F}$ & - & - & - & - & - & - \\
\hline & $\varphi$ & - & - & - & - & - & - \\
\hline \multirow{2}{*}{$\begin{array}{l}\text { Diferencias significativas } \\
\text { entre grupos: Especialistas } \\
\text { con menos de } 10 \text { años de } \\
\text { experiencia y Especialistas con } \\
\text { más de } 20 \text { años de experiencia }\end{array}$} & $\mathrm{F}$ & $-3 \cdot 36$ & - & - & - & - & - \\
\hline & $\varphi$ & .045 & - & - & - & - & - \\
\hline \multirow{2}{*}{$\begin{array}{l}\text { Diferencias significativas entre } \\
\text { grupos: Especialistas con } \\
\text { 10-20 años de experiencia y } \\
\text { Especialistas con más de } 20 \\
\text { años de experiencia }\end{array}$} & $\mathrm{F}$ & -3.12 & -3.04 & -3.26 & - & - & -2.17 \\
\hline & $\varphi$ & .017 & .033 & .017 & - & - & .035 \\
\hline
\end{tabular}

Fuente: Elaboración propia, 2021.

Nota: M - media aritmética; SD - desviación de la media cuadrada; F - la diferencia promedio; $\varphi$ valor; destacamos diferencias significativas en negrita y diferencias a nivel de la tendencia estadística en cursiva.

En general, en la muestra, los indicadores de percepción subjetiva del pasado de los especialistas que se encuentran en la etapa de desarrollo de la conciencia profesional, están dentro del intervalo característico para la muestra normativa, acercándose al límite superior. Las puntuaciones más 
Ihor Popovych, Diego Felipe Arbeláez-Campillo, Magda Julissa Rojas-Bahamón, Iryna Burlakova, Vitaliy Kobets y Halyna Bokshan

altas se han registrado en las escalas: "Coloración emocional del pasado" y "Magnitud del pasado", y las más bajas, en las escalas: "Estructura del pasado" y "Sentido del pasado". Se observa una tendencia similar en cada grupo profesional por separado.

Se han encontrado diferencias significativas $(\mathrm{p}<.05)$ entre los representantes de grupos profesionales de encuestados "Especialistas con menos de 10 años de experiencia" y "Especialistas con más de 20 años de experiencia", así como "Especialistas con experiencia de 10 a 20 años" y "Especialistas con experiencia de más de 20 años" en la escala de "Magnitud del pasado": en ambos casos los indicadores son superiores en los especialistas con más de 20 años de experiencia. Es decir, con mayor frecuencia que los otros, los encuestados con más de 20 años de experiencia evalúan su pasado como lleno de significado y experiencias (en su mayoría positivas), lo que da espacio para la autorrealización y generalmente coincide con los datos obtenidos a través de cuestionarios "ZTPI" y "TFTPI" (Zimbardo y Bojd, 2010).

Se han encontrado diferencias significativas $(\mathrm{p}<.05)$ entre los representantes de grupos profesionales "Especialistas con 10-20 años de experiencia" y "Especialistas con más de 20 años de experiencia" en los indicadores de las escalas "Actividad del pasado", "Coloración emocional del pasado" y evaluación promedio de factores pasados: son superiores en especialistas con más de 20 años de experiencia. Es decir, los encuestados con más de 20 años de experiencia tienden a percibir su pasado como enérgico, posiblemente debido a un cierto estrés emocional y una alta tasa de eventos, así como a evaluar sus emociones pasadas como positivas. Pueden estar más satisfechos con la situación actual de la vida en el pasado.

Vamos a examinar las características de la percepción subjetiva del presente en representantes de diferentes grupos profesionales por el método del "Diferencial Semántico del Tiempo" ("DST") (Tabla 4). 
Tabla 4. Indicadores de percepción subjetiva del presente en diversos grupos profesionales de especialistas en el área económica de acuerdo con "SDT"

\begin{tabular}{|c|c|c|c|c|c|c|c|}
\hline \multirow{2}{*}{\multicolumn{2}{|c|}{ Escala }} & \multicolumn{6}{|c|}{ Parámetros } \\
\hline & & \multirow[t]{2}{*}{ 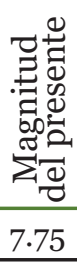 } & \multirow[t]{2}{*}{ 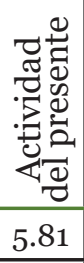 } & \multirow[t]{2}{*}{ 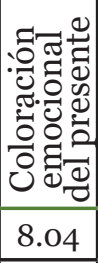 } & \multirow{2}{*}{ 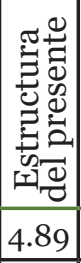 } & \multirow[t]{2}{*}{ 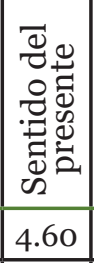 } & \multirow[t]{2}{*}{ 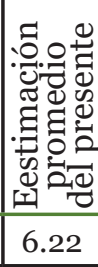 } \\
\hline \multirow{2}{*}{$\begin{array}{l}\text { Desarrollo de la conciencia } \\
\text { profesional }\end{array}$} & M & & & & & & \\
\hline & SD & 4.67 & 4.56 & 4.76 & 4.87 & 3.82 & 3.03 \\
\hline \multirow{2}{*}{$\begin{array}{l}\text { Especialistas con menos de } 10 \\
\text { años de experiencia }\end{array}$} & M & 6.00 & 3.83 & 5.39 & $5 \cdot 39$ & 2.43 & 4.60 \\
\hline & SD & 7.19 & 4.57 & 5.53 & 5.81 & 3.69 & 3.58 \\
\hline \multirow{2}{*}{$\begin{array}{l}\text { Especialistas con 10-20 años de } \\
\text { experiencia }\end{array}$} & $\mathrm{M}$ & 8.00 & 5.63 & 8.34 & 4.34 & 4.85 & 6.24 \\
\hline & SD & 3.68 & 4.83 & 4.33 & 4.80 & 3.92 & 2.90 \\
\hline \multirow{2}{*}{$\begin{array}{l}\text { Especialistas con más de } 20 \\
\text { años de experiencia }\end{array}$} & M & 8.64 & 7.82 & 9.57 & 5.71 & 5.82 & 7.51 \\
\hline & SD & 3.78 & 3.02 & 4.26 & 4.14 & 3.03 & 2.19 \\
\hline \multirow{2}{*}{$\begin{array}{l}\text { Diferencias significativas entre } \\
\text { grupos: Especialistas con menos } \\
\text { de } 10 \text { años de experiencia y } \\
\text { Especialistas con 10-20 años de } \\
\text { experiencia }\end{array}$} & $\mathrm{F}$ & - & - & -2.95 & - & $-2.42 \mid$ & $\mid-1.64$ \\
\hline & $\varphi$ & - & - & .027 & - & .021 & .059 \\
\hline \multirow{2}{*}{$\begin{array}{l}\text { Diferencias significativas entre } \\
\text { grupos: Especialistas con menos } \\
\text { de 10 años de experiencia y } \\
\text { Especialistas con más de } 20 \\
\text { años de experiencia }\end{array}$} & $\mathrm{F}$ & - & -3.99 & -4.18 & - & $\mid-3 \cdot 39$ & $|-2.91|$ \\
\hline & $\varphi$ & - & .004 & .005 & - & .004 & .001 \\
\hline \multirow{2}{*}{$\begin{array}{l}\text { Diferencias significativas entre } \\
\text { grupos: Especialistas con } \\
\text { 10-20 años de experiencia y } \\
\text { Especialistas con más de } 20 \\
\text { años de experiencia }\end{array}$} & $\mathrm{F}$ & - & -2.19 & - & - & - & - \\
\hline & $\varphi$ & - & .076 & - & - & - & - \\
\hline
\end{tabular}

Fuente: Elaboración propia, 2021.

Nota: M - media aritmética; SD - desviación de la media cuadrada; F - la diferencia promedio; $\varphi$ valor; destacamos diferencias significativas en negrita y diferencias a nivel de la tendencia estadística en cursiva.

En una muestra de profesionales que se encuentran en la etapa de desarrollo de la conciencia profesional, los indicadores de percepción subjetiva del presente están dentro de la norma, acercándose a su límite superior, a excepción del indicador "Coloración emocional del presente". 
Ihor Popovych, Diego Felipe Arbeláez-Campillo, Magda Julissa Rojas-Bahamón, Iryna Burlakova, Vitaliy Kobets y Halyna Bokshan

En esta escala se registran las puntuaciones sobreestimadas. En general, las puntuaciones más altas son observadas en las escalas: "Coloración emocional del presente" y "La magnitud del presente", así como las puntuaciones más bajas, en las escalas: "Sentido del presente" y "Estructura del presente". Una tendencia similar se observa en los grupos "Especialistas con 10-20 años de experiencia" y "Especialistas con más de 20 años de experiencia”. Sin embargo, en el grupo "Especialistas con menos de 10 años de experiencia" predomina "Magnitud del presente", y las puntuaciones más bajas se observan en las escalas "Sentido del tiempo presente" y "Actividad del presente".

Se han encontrado diferencias significativas $(\mathrm{p}<.05)$ entre los representantes de grupos profesionales de encuestados "Especialistas con menos de 10 años de experiencia" y "Especialistas con más de 20 años de experiencia", así como diferencias a nivel de tendencia estadística $(\mathrm{p}<.01)$ entre los representantes de grupos profesionales "Especialistas con 10-20 años de experiencia" y "Especialistas con más de 20 años de experiencia" en los indicadores de las escalas "Actividad del presente". En todos los casos, son superiores en los especialistas con más de 20 años de experiencia. Es decir, los profesionales que tienen más de 20 años de experiencia, más que otros profesionales, tienden a considerar sus vidas como enérgicas, con una alta tasa de eventos.

Se han encontrado diferencias significativas $(\mathrm{p}<.05)$ entre los representantes de grupos profesionales de encuestados "Especialistas con menos de 10 años de experiencia" y "Especialistas con 10-20 años de experiencia", diferencias significativas ( $\mathrm{p}<.01$ ) entre los grupos estudiados en los indicadores de las escalas: "Coloración emocional del presente" y "Sentido del presente". Así como diferencias a nivel de tendencia estadística $(\mathrm{p}<.01)$ entre los grupos "Especialistas con menos de 10 años de experiencia" y "Especialistas con experiencia de10 a 20 años" y diferencias significativas $(\mathrm{p}<.01)$ entre los grupos "Especialistas con menos de 10 años de experiencia" y "Especialistas con más de 20 años de experiencia”. Según la evaluación promedio de los factores del presente: todos estos indicadores son más bajos en los encuestados con menos de 10 años de experiencia. Están menos inclinados a evaluar su situación de vida actual como algo agradable para ellos, a sentir experiencias, emociones y sentimientos vívidos sobre su presente; sentir la propia implicación emocional en los acontecimientos de la vida; sentirse un participante activo en lo que está sucediendo.

Vamos a examinar las características de la percepción subjetiva del futuro en representantes de diferentes grupos profesionales por el método del "Diferencial Semántico del Tiempo" ("DST") (ver Tabla 5). 
Tabla 5. Indicadores de percepción subjetiva del futuro en diversos grupos profesionales de especialistas en el área económica de acuerdo con "SDT"

\begin{tabular}{|c|c|c|c|c|c|c|c|}
\hline \multirow{2}{*}{ Escala } & & \multicolumn{6}{|c|}{ Parámetros } \\
\hline & & \multirow{2}{*}{ 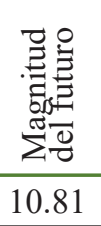 } & \multirow{2}{*}{ 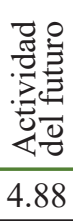 } & \multirow{2}{*}{ 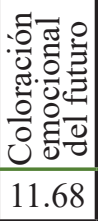 } & \multirow{2}{*}{ 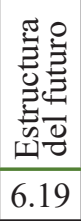 } & \multirow{2}{*}{ 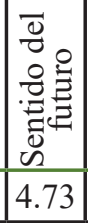 } & \multirow{2}{*}{ 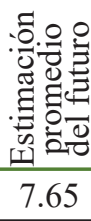 } \\
\hline \multirow{2}{*}{$\begin{array}{l}\text { Desarrollo de la conciencia } \\
\text { profesional }\end{array}$} & $\mathrm{M}$ & & & & & & \\
\hline & SD & 3.50 & 3.84 & 2.54 & 5.43 & 5.19 & 2.58 \\
\hline \multirow{2}{*}{$\begin{array}{l}\text { Especialistas con menos de } 10 \\
\text { años de experiencia }\end{array}$} & $\mathrm{M}$ & 11.52 & 5.74 & 11.26 & 7.00 & 5.26 & 8.14 \\
\hline & SD & 3.03 & 4.28 & 2.28 & 4.44 & 4.54 & 2.37 \\
\hline \multirow{2}{*}{$\begin{array}{l}\text { Especialistas con 10-20 años de } \\
\text { experiencia }\end{array}$} & $\mathrm{M}$ & 10.32 & 5.21 & 12.00 & 5.32 & 5.08 & 7.59 \\
\hline & SD & 3.88 & 3.64 & 2.54 & 6.21 & 5.41 & 2.74 \\
\hline \multirow{2}{*}{$\begin{array}{l}\text { Especialistas con más de } 20 \text { años } \\
\text { de experiencia }\end{array}$} & $\mathrm{M}$ & 11.29 & 3.46 & 11.32 & 7.43 & 3.50 & 7.40 \\
\hline & $\mathrm{SD}$ & 2.81 & 3.65 & 2.74 & 3.90 & 5.18 & 2.43 \\
\hline \multirow{2}{*}{$\begin{array}{l}\text { Diferencias significativas entre } \\
\text { grupos: Especialistas con menos } \\
\text { de } 0 \text { años de experiencia y } \\
\text { Especialistas con } 10-20 \text { años de } \\
\text { experiencia }\end{array}$} & $\mathrm{F}$ & - & - & - & - & - & - \\
\hline & $\varphi$ & - & - & - & - & - & - \\
\hline \multirow{2}{*}{$\begin{array}{l}\text { Diferencias significativas entre } \\
\text { grupos: Especialistas con menos } \\
\text { de } 0 \text { años de experiencia y } \\
\text { Especialistas con más de } 20 \text { años } \\
\text { de experiencia }\end{array}$} & $\mathrm{F}$ & - & 2.28 & - & - & - & - \\
\hline & $\varphi$ & - & .095 & - & - & - & - \\
\hline \multirow{2}{*}{$\begin{array}{l}\text { Diferencias significativas } \\
\text { entre grupos: Especialistas con } \\
10-20 \text { años de experiencia y } \\
\text { Especialistas con más de } 20 \text { años } \\
\text { de experiencia }\end{array}$} & $\mathrm{F}$ & - & - & - & - & - & - \\
\hline & $\varphi$ & - & - & - & - & - & - \\
\hline
\end{tabular}

Fuente: Elaboración propia, 2021.

Nota: M - media aritmética; SD - desviación de la media cuadrada; F - la diferencia promedio; $\varphi$ valor; destacamos diferencias significativas en negrita y diferencias a nivel de la tendencia estadística en cursiva.

Se establece que en los especialistas que se encuentran en la etapa de desarrollo de la conciencia profesional, los indicadores de percepción subjetiva del futuro se encuentran dentro del intervalo normativo, acercándose a su límite superior, excepto los indicadores "Coloración emocional del futuro", "Magnitud del futuro" y "Estimación promedio del futuro": las puntuaciones de estas características están sobreestimados. 
Ihor Popovych, Diego Felipe Arbeláez-Campillo, Magda Julissa Rojas-Bahamón, Iryna Burlakova, Vitaliy Kobets y Halyna Bokshan

En general, las puntuaciones más altas en la muestra se observan en las escalas: "Coloración emocional del futuro" y "Magnitud del futuro", así como las puntuaciones más bajas, en las escalas: "Sentido del futuro" y "Actividad del futuro". Se observa una tendencia similar en todos los grupos por separado.

Se han encontrado diferencias a nivel de tendencia estadística $(\mathrm{p}<.01)$ entre los representantes de los grupos profesionales "Especialistas con menos de 10 años de experiencia" y "Especialistas con más de 20 años de experiencia" en la escala "Actividad futura": estas diferencias son más altas en los encuestados con menos de 10 años de experiencia. Son más propensos que los especialistas con más de 20 años de experiencia a percibir su futuro como enérgico, pasando a un ritmo alto y lleno de acontecimientos. Se debe notar que estos datos coinciden con los datos obtenidos a través de cuestionarios “ZTPI” y “TFTPI” (Zimbardo y Bojd, 2010).

Vamos a considerar las características de la percepción subjetiva del en diferentes grupos profesionales de especialistas en el área económico, teniendo en cuenta el factor de género por el método del "Diferencial Semántico del Tiempo" ("DST") (Tabla 6).

Tabla 6. Indicadores de percepción subjetiva del tiempo por los especialistas en el área económica teniendo en cuenta el género de los encuestados de acuerdo con "SDT"

\begin{tabular}{|l|c|c|c|c|c|c|}
\hline \multirow{2}{*}{ Escala } & \multicolumn{2}{l|}{ Hombres } & \multicolumn{2}{l|}{ Mujeres } & \multicolumn{2}{l}{$\begin{array}{l}\text { Valores de las } \\
\text { diferencias }\end{array}$} \\
\cline { 2 - 8 } & $\mathbf{M}$ & SD & M & SD & F & $\boldsymbol{\varphi}$ \\
\hline Magnitud del presente & 7.72 & 4.73 & 7.78 & 4.67 & - & - \\
\hline Actividad del presente & 4.91 & 4.36 & 6.60 & 4.62 & - & - \\
\hline $\begin{array}{l}\text { Coloración emocional del } \\
\text { presente }\end{array}$ & 7.85 & 4.88 & 8.22 & 4.69 & - & - \\
\hline Estructura del presente & 5.45 & 4.75 & 4.40 & 4.96 & 3.33 & .071 \\
\hline Sentido del presente & 3.38 & 3.58 & 5.68 & 3.74 & 7.56 & .007 \\
\hline $\begin{array}{l}\text { Estimación promedio del } \\
\text { presente }\end{array}$ & 5.86 & 2.99 & 6.53 & 3.06 & - & - \\
\hline Magnitud del pasado & 5.58 & 5.66 & 7.03 & 4.80 & - & - \\
\hline Actividad del pasado & 3.70 & 4.68 & 4.02 & 5.78 & - & - \\
\hline $\begin{array}{l}\text { Coloración emocional del } \\
\text { pasado }\end{array}$ & 8.21 & 4.36 & 7.25 & 5.66 & - & - \\
\hline
\end{tabular}




\begin{tabular}{|l|c|c|c|c|c|c|}
\hline Estructura del pasado & .79 & 5.17 & 1.98 & 5.00 & - & - \\
\hline Sentido del pasado & 1.87 & 3.92 & 1.55 & 5.51 & - & - \\
\hline $\begin{array}{l}\text { Estimación promedio del } \\
\text { pasado }\end{array}$ & 4.03 & 3.48 & 4.37 & 4.00 & - & - \\
\hline Magnitud del futuro & 10.36 & 3.49 & 11.20 & 3.48 & - & - \\
\hline Actividad del futuro & 4.51 & 4.41 & 5.22 & 3.25 & - & - \\
\hline $\begin{array}{l}\text { Coloración emocional del } \\
\text { futuro }\end{array}$ & 11.11 & 2.33 & 12.18 & 2.64 & - & - \\
\hline Estructura del futuro & 5.47 & 5.84 & 6.82 & 50.00 & - & - \\
\hline Sentido del futuro & 5.66 & 4.59 & 3.90 & 5.58 & $\mathbf{4 . 1 2}$ & $\mathbf{. 0 4 5}$ \\
\hline $\begin{array}{l}\text { Estimación promedio del } \\
\text { futuro }\end{array}$ & 7.42 & 2.71 & 7.86 & 2.47 & - & - \\
\hline
\end{tabular}

Fuente: Elaboración propia, 2021.

Nota: $\mathrm{M}$ - media aritmética; SD - desviación de la media cuadrada; F - la diferencia promedio; $\varphi$ valor; destacamos diferencias significativas en negrita y diferencias a nivel de la tendencia estadística en cursiva.

Los resultados del análisis de varianza mostraron diferencias significativas $(p<.01)$ entre hombres y mujeres en la escala: "Sentido del presente" (más pronunciado en las mujeres); diferencias significativas ( $p<.05)$ en la escala "Sentido del futuro" (más pronunciado en hombres); y diferencias a nivel de tendencia estadística en el indicador "Estructura del presente" (más pronunciado en hombres).

\section{Discusión}

Se ha determinado que los especialistas con más de 20 años de experiencia están más enfocados en el pasado y el presente, y los que tienen menos de 10 años de experiencia están enfocados en el futuro ( $\mathrm{p} \leq .01)$. En la mayoría de los casos, los especialistas con más de 20 años de trabajo tienen una rica experiencia de vida, una carrera exitosa que recuerdan con orgullo; su posición implica una participación activa en la vida de su organización, en la toma de decisiones; el futuro les preocupa menos que a otros profesionales que solo pueden prever una carrera exitosa en el futuro.

Los profesionales con 10-20 años de experiencia a menudo entran en contacto con nuevas personas, tienen que resolver situaciones de conflicto, trabajar en un entorno en constante cambio y, si es posible, buscan evaluar de antemano los posibles riesgos y posibles problemas; los profesionales con menos de 10 años de experiencia a menudo planean su futuro y buscan el apoyo de personas más experimentadas y relevantes. 
Ihor Popovych, Diego Felipe Arbeláez-Campillo, Magda Julissa Rojas-Bahamón, Iryna Burlakova, Vitaliy Kobets y Halyna Bokshan

Los resultados del estudio muestran que los hombres son más propensos que las mujeres a ocupar puestos de liderazgo y tienen el tiempo y los recursos para desarrollar sus actividades profesionales. Ellos prestan menos atención a las manifestaciones de enfermedades y diversas dolencias y por eso en el cuestionario de autoevaluación ponen puntuaciones altas en el estado de salud y otros indicadores. Se ha descubierto que los hombres tienen una orientación temporal más pronunciada para el "Futuro" y las mujeres, para el "Futuro Trascendente" $(\mathrm{p} \leq .05)$. Las mujeres son más propensas a la inmersión emocional en los eventos actuales, mientras que los hombres son más propensos a estructurar los eventos actuales y planificar eventos futuros ( $\mathrm{p} \leq .05)$. Creemos que esto está en línea con ciertos hechos y estereotipos de género: los hombres están más centrados y son capaces de estructurar los eventos, y las mujeres están más inclinadas a los ritos religiosos, más a menudo recurren a astrólogos, horóscopos, buscan ciertos "signos" dados por el universo, etc. Los hombres son más propensos a la planificación estratégica, la reflexión y la superación preventiva que las mujeres $(\mathrm{p} \leq .05)$. Quizás esto esté en línea con la opinión pública: los hombres son considerados los mejores estrategas, capaces de evaluar todos los riesgos de antemano y tomar las medidas preventivas necesarias, y las mujeres prefieren actuar en función de la situación.

\section{Conclusiones}

Se ha establecido que las personas que ocupan puestos de liderazgo tienen más tiempo, oportunidades y recursos para desarrollar sus actividades profesionales. Tienen menos tareas que los deprimen y los irritan, pues éstas pueden delegarse, posponerse o cancelarse, lo que tiene un efecto positivo en su estado psicológico. Los especialistas con más de 20 años de experiencia tienen un cierto estatus social, lo que les otorga privilegios especiales que reflejan su autoridad, competencia profesional, importancia para los demás y afecta también a las condiciones de trabajo y los salarios.

Es decir, las mujeres son más propensas a sentir su propia participación emocional e intelectual en los acontecimientos del presente que los hombres. Al mismo tiempo, los hombres son más propensos que las mujeres a presentarse como participantes activos en los eventos futuros, a sentir su propia importancia para los acontecimientos del futuro; y con mayor frecuencia evalúan su presente como estructurado, predecible y comprensible. Por lo tanto, ellos entienden la relación entre los eventos actuales y perciben el mundo como más estable y seguro que las mujeres.

El problema de estudiar el tiempo psicológico del individuo es uno de los menos desarrollados en la psicología mundial. Creemos que los resultados 
obtenidos pueden utilizarse en asesoramiento psicológico, evaluación del personal (orientación profesional, selección del personal, reclutamiento). Y también servirán como base para la creación de mantenimiento metódico para una estimación de posibles problemas de actividad de los especialistas en el área económica en puestos concretos y el desarrollo de formas de resolver estos problemas.

\section{Referencias Bibliográficas}

ACEVEDO-DUQUE, Ángel; ARGÜELLO, Alejandro; PINEDA, Bessy; TURCIOS, Paola. 2020. "Competencias del docente en educación online en tiempo de COVID-19: Universidades Públicas de Honduras". En: Revista de Ciencias Sociales (Ve). Vol. XXVI, No. especial 2, pp. 206-224.

ALLEN, David. 2002. "Getting things done: the art of stress-free productivity". In: Penguin Books. New York, USA.

ARBELÁEZ-CAMPILLO, Diego Felipe; ROJAS-BAHAMÓN, Magda Julissa; ARBELÁEZ-ENCARNACIÓN, Tanya. 2018. "Apuntes para el debate de las categorías ciudadanía universal, derechos humanos y globalización" En: Cuestiones Políticas. Vol 34, No. 61, pp. 139-160.

BLYNOVA, Olena; CHERVINSKA, Inna; KAZIBEKOVA, Viktoriia; BOKSHAN, Halyna; YAKOVLEVA, Svitlana; ZAVERUKHA, Olha; POPOVYCH, Ihor. 2020a. "Social and Psychological Manifestations of Professional Identity Crisis of Labor Migrants". In: Revista Inclusiones. Vol 7, No. 3, pp. 93-105. Disponible en línea. En: http://www. revistainclusiones.org/index.php/inclu/article/view/1318. Fecha de consulta: 14/09/2020.

BLYNOVA, Olena; KRUGLOV, Konstantyn. 2019. "The value of social capital for the psychological well-being of employees". In: Insight: the psychological dimensions of society. No. 1, pp. 72-78. Disponible en línea. En: https://doi.org/10.32999/2663-970X/2019-1-11. Fecha de consulta: 14/09/2020.

BLYNOVA, Olena; LAPPO, Violetta; KALENCHUK, Valentyna; AGARKOV, Oleg; SHRAMKO, Ihor; LYMARENKO, Lidiia; POPOVYCH, Ihor. 202ob. "Corporate Culture of a Higher Education Institution as a Factor in Forming Students' Professional Identity". En: Revista Inclusiones. Vol. 7, No. (Especial), pp. 481-496. Disponible en línea. En: http://www.revistainclusiones.org/index.php/inclu/article/ view/1305. Fecha de consulta: 14/09/2020. 
Ihor Popovych, Diego Felipe Arbeláez-Campillo, Magda Julissa Rojas-Bahamón, Iryna Burlakova, Vitaliy Kobets y Halyna Bokshan

BLYNOVA, Olena; MOISEIENKO, Viktoriia; LOS, Oksana; BURLAKOVA, Iryna; YEVDOKIMOVA, Olena; TOBA, Marianna; POPOVYCH, Ihor. 2020c. "Assertiveness as a Factor of Students' Choice of Behavior Strategies in Social Interaction”. En: Revista Inclusiones. Vol. 7, No. 4, pp. 259-272. Disponible en línea. En: http://www.revistainclusiones. org/index.php/inclu/article/view/1551. Fecha de consulta: 14/11/2020.

BLYNOVA, Olena; POPOVYCH, Ihor; SEMENOVA, Nataliia; KASHYRINA, Yevheniia; URSULENKO, Olena; KONONENKO, Okana. 202Od. "Personality Factors of Choosing Adaptation Strategies in a Different Cultural Environment by Labor Migrants from Ukraine”. En: Revista Amazonia Investiga. Vol 9, No. 32, pp. 45-54. Disponible en línea. En: http://dx.doi.org/10.34069/AI/2020.32.08.5. Fecha de consulta: 14/06/2020.

BOLOTOVA, Alla. 2012. "Time and space in interpersonal relationships" In: Journal of the Higher School of Economics. Vol 9, No. 3, pp. 22-36.

CRUZ DEL CARMEN, Eglis. 2019. "Importancia del manejo de competencias tecnológicas en las prácticas docentes de la Universidad Nacional Experimental de la Seguridad (UNES)” En: Revista Educación. Vol 43, No. 1, pp. 196-218. Disponible en línea. En: https://doi.org/10.15517/ revedu.v43i1.27120. Fecha de consulta: 14/06/2020.

FERNÁNDEZ-RIVAS, Maria; ESPADA-MATEOS, María. 2019. "The knowledge, continuing education and use of teaching styles in Physical Education teachers" In: Journal of Human Sport and Exercise. Vol 14, No. 1, pp. 99-111. Disponible en línea. En: https://doi.org/10.14198/ jhse.2019.141.08. Fecha de consulta: 14/06/2020.

HALIAN,Andriy; HALIAN, Ihor; BURLAKOVA, Iryna; SHEVCHENKO, Rosina; LAPPO, Violetta; ZHIGARENKO, Igor; POPOVYCH, Ihor. 2020 a. "Emotional Intelligence in the Structure of Adaptation Process of Future Healthcare Professionals" In: Revista Inclusiones. Vol 7, No. 3, pp. 447460. Disponible en línea. En: http://www.revistainclusiones.org/index. php/inclu/article/view/1347. Fecha de consulta: 14/06/2020.

HALIAN, Ihor; HALIAN, Olena; GUSAK, Liudmyla; BOKSHAN, Halyna; POPOVYCH, Ihor. 2020b. Communicative Competence in Training Future Language and Literature Teachers. In: Revista Amazonia Investiga. Vol 9, No. 29, pp. 530-541. Disponible en línea. En: https:// amazoniainvestiga.info/index.php/amazonia/article/view/1417. Fecha de consulta: 14/06/2020.

HALIAN, Ihor; MACHYNSKA, Natalia; LOZYNSKA, Svitlana; NOS, Liubov; DERKACH, Yuliya; PROTS, Marta; POPOVYCH, Ihor. 2020c. 
Tolerance of uncertainty as a component of the process of life-creation of future educators. Revista Inclusiones. Vol 7, No. Especial, pp. 512-528. Disponible en línea. En: http://www.revistainclusiones.org/index.php/ inclu/article/view/1307. Fecha de consulta: 14/06/2020.

JAMA NETWORK. 2013. "World medical association declaration of Helsinki" En: Ethical principles for medical research involving human subjects. Vol. 310, No. 20, pp. 2191-4. Disponible en línea. En: https://doi. org/10.1001/jama.2013.281053. Fecha de consulta: 14/06/2020.

KALENCHUK, Valentyna Oleksandrivna. 2019. "Student's Social Status as a Factor of Assessment of the University's Organizational Culture" In: The psychological dimensions of society. No. 2, pp. 72-77. Disponible en línea. En: https://doi.org/10.32999/2663-970X/2019-2-10. Fecha de consulta: 12/12/2020.

KHMILIAR, Oleh; POPOVYCH, Ihor; HRYS, Antonina; PAVLIUK, Mariya: ZAVATSKA, Nataliia; LYTVYNENKO, Olga; BLYNOVA, Olena. 2020. "Spatial Regulation of Personality Behavior in the Conditions of Progression of the COVID-19 Pandemic" In: Revista Inclusiones. Vol. 7. No. (Especial), pp. 289-306. Disponible en línea. En: http://www. revistainclusiones.org/index.php/inclu/article/view/176o. Fecha de consulta: 12/12/2020.

KOBETS, Vitaliy; POLTORATSKIY, Maksim. 2014. "Forming an evolutionarily stable firm strategy under Cournot competition using social preferences" In: Communications in Computer and Information Science. Vol. 469, pp. 343-361.

KOBETS, Vitaliy; YATSENKO, Valeria. 2016. "Adjusting business processes by the means of an autoregressive model using BPMN 2.o." In: CEUR Workshop Proceedings. No. 1614, pp. 518-533.

KONONENKO, Oksana; KONONENKO, Anatoliy; STYNSKA, Viktoriia; KACHMAR, Oleksandra; PROKOPIV, Liubov; KATOLYK, Halyna; POPOVYCH, Ihor. 2020. Research of the factor structure of the model of world view settings at a young age. In: Revista Inclusiones. Vol 7, No. 3, pp. 98-116. Disponible en línea. http://www.revistainclusiones.org/ index.php/inclu/article/view/1618. Fecha de consulta: 13/06/2020.

KRUPNYK, Ivan Romanovych; TKALENKO, Natalya Viktorivna. 2019. "Manipulative Behavior in the Professional Activities of Office Staff" In: Insight: the psychological dimensions of society. Vol 1, pp. 96-101. En: https://doi.org/10.32999/2663-970X/2019-1-15. Fecha de consulta: 13/06/2020. 
Ihor Popovych, Diego Felipe Arbeláez-Campillo, Magda Julissa Rojas-Bahamón, Iryna Burlakova, Vitaliy Kobets y Halyna Bokshan

MOYA, Fabio; LÓPEZ, Danny Daniel; ARTIGAS, Wileidys. 2018. “Herramientas gerenciales desde el enfoque socio crítico en universidades: Estudio exploratorio en Venezuela y Colombia" En: Revista de Ciencias Sociales (Ve). Vol. XXIV, No. 1, pp. 101-112.

NOSOV, Pavlo; PALAMARCHUK, Ihor; ZINCHENKO, Serhii; POPOVYCH, Ihor; NAHRYBELNYI, Yaroslav; NOSOVA Hanna. 2020a."Development of means for experimental identification of navigator attention in ergatic systems of maritime transport" In: Bulletin of University of Karaganda. Vol 1, No. 97, pp. 58-69.

NOSOV, Pavlo; ZINCHENKO, Serhii; POPOVYCH, Ihor; BEN, Andrew; NAHRYBELNYI, Yaroslav; MATEICHUK, Vadym. 202ob. "Diagnostic system of perception of navigation danger when implementation complicated maneuvers" In: Electronics, Computer Science, Control. No. 1, pp. 146-161.

NUTTIN, Joseph. 2004. Motivation, action, and future perspective. Smysl. Moscow, Russia.

PLOKHIKH, Viktor. 2006. "The accuracy of the subjective assessment of the time limits of activity as a factor in the success of solving the tracking task” In: Psychological Journal. Vol 26, No. 2, pp. 93-101.

PINKOVETSKAIA, Iuliia; ARBELÁEZ-CAMPILLO, Diego Felipe; ROJASBAHAMÓN, Magda Julissa; VEAS INIESTA, Daniela. 2020. "Motivation of new entrepreneurs in modern economies" En. Amazonia Investiga. Vol 9, No. 29, pp. 368-373. Disponible en línea. En: https://doi. org/10.34069/AI/2020.29.05.41. Fecha de consulta: 14/12/2020.

POPOVYCH, Ihor; ZHIGARENKO, Igor; LOSIYEVSKA, Olga; DOVBENKO, Svitlana; KASHYRINA, Yevheniia; SHEVCHENKO, Rosina; PILETSKA, Liybomyra. 2020. "Research of Achievement Motivation's Impaction the Career Orientations of Future Managers of Organization" In: Revista Inclusiones. Vol. 7, No. (Especial), pp. 247-263. Disponible en línea. En: http://www.revistainclusiones.org/index.php/inclu/article/view/1231. Fecha de consulta: 14/06/2020.

PROKONICH, Oleg. 2014. "The relationship of the characteristics of the time perspective of the personality of university students with the semantic aspects of the attitude to the learning process" In: Bulletin of KemSU. Vol 4, No. 60, pp. 117-122.

ROMERO-ARGUETA, José de Jesús; COREAS-FLORES, Edwin Osmil; SEVERINO-GONZÁLEZ, Pedro. 2020. "Responsabilidad social del estudiante universitario en El Salvador: Género y territorio" En: Revista de Ciencias Sociales (Ve). Vol XXVI, No. 2, pp. 426-441. 
SHUL'C, Duane; SHUL'C, Sydney. 2002. History of modern psychology. Evrazija. St. Petersburg, Russia.

SHEVCHENKO, Rosina; POPOVYCH, Ihor; SPYTSKA, Liana; NOSOV, Pavlo; ZINCHENKO, Serhii; MATEICHUK, Vadym; BLYNOVA, Olena. 2020. "Comparative analysis of emotional personality traits of the students of maritime science majors caused by long-term staying at sea" In: Revista Inclusiones. Vol. 7, No. (Especial), pp. 538-554. Disponible en línea. En: http://www.revistainclusiones.org/index.php/inclu/article/view/1309. Fecha de Consulta: 14/06/2020.

TSIUNIAK, Oksana; PYSLAR, Anatolii; LIALIUK, Galina; BONDARENKO, Viktoriia; KOVTUN, Oksana; LOS, Oksana; POPOVYCH, Ihor. 2020. "Research of interdependence of variables and factor structure of masters' readiness for innovative pedagogical activity" En: Revista Inclusiones. Vol 7, No. 3, pp. 427-452. Disponible en línea. En: http:// www.revistainclusiones.org/index.php/inclu/article/view/1645. Fecha de Consulta: 14/09/2020.

VARGUILLAS, Carmen Siavil; BRAVO, Patricia Cecilia. 2020. "Virtualidad como herramienta de apoyo a la presencialidad: Análisis desde la mirada estudiantil” En: Revista de Ciencias Sociales (Ve). Vol XXVI, No. 1, pp. 219- 232.

VASSERMAN, Ludwig; TRIFONOVA, Elena; CHERVINSKAJA, Ksenia. 2009. Semantic time differential: expert psychodiagnostic system in medical psychology. Piter. St. Petersburg, Russia.

ZIMBARDO, Philip; BOJD, John. 2010. The Time Paradox: The New Psychology of Time That Will Change Your Life. In: Rech. St. Petersburg, Russia.

ZINCHENKO, Serhii; BEN, Andrew; NOSOV, Pavlo; POPOVYCH, Ihor; MAMENKO, Pavlo; MATEYCHUK, Vadym. 2020. "Improving the accuracy and reliability of automatic vessel moution control systems" En: Radio Electronics, Computer Science, Control. No 2, pp. 183-195. Disponible en línea. En: DOI: 10.15588/1607-3274-2020-2-19. Fecha de consulta: 12/19/2020.

ZINCHENKO, Serhii; NOSOV, Pavlo; MATEICHUK, Vadym; MAMENKO, Pavlo; Popovych, Ihor; Grosheva, Olga. 2019. "Automatic collision avoidance system with many targets, including maneuvering ones" En: Bulletin of the University of Karaganda - Physics. Vol 4, No. 96, pp. 6979. Disponible en línea. En: DOI: 10.31489/2019Ph4/69-79. Fecha de consulta: 12/19/2020. 
Vol. 39 N $^{\circ} 69$

Esta revista fue editada en formato digital y publicada en julio de 2021, por el Fondo Editorial Serbiluz, Universidad del Zulia. Maracaibo-Venezuela 